\section{Análise de redes na regulação do tratamento do câncer do aparelho digestivo}

\author{
Network analysis of the regulation of \\ gastrointestinal cancer treatment
}

\section{Análisis de redes en la regulación del tratamiento del cáncer del aparato digestivo}

Cibele Barbosa Carroll 1,2

Marcia Gomide 1

doi: 10.1590/0102-311X00041518

\section{Resumo}

A alta complexidade é componente fundamental da Politica Nacional para a Prevenção e Controle do Câncer no âmbito do Sistema Único de Saúde (SUS) brasileiro. Tem como obrigação garantir cuidado integral aos pacientes. A regulação é parte da estrutura organizacional, sendo responsável por definir os fluxos de atendimento. No Rio de Janeiro, a Central de Regulação iniciou as atividades em junho de 2015, organizando procedimentos ambulatoriais de alta complexidade. O presente trabalho tem como objetivo analisar o deslocamento para o tratamento de pessoas com tumores digestivos no Estado do Rio de Janeiro, antes e após a atuação da regulação, sob a perspectiva da Análise de Redes Sociais (ARS). Foi desenvolvido um estudo ecológico, comparando os períodos anterior (2013) e posterior (2016) à implantação da central reguladora. A pesquisa foi desenvolvida com a utilização de dados secundários provenientes do Departamento de Informática do SUS. Desse modo, foram desenhados dois sociogramas referentes aos anos 2013 e 2016, relacionando local de residência com local de internação. Com essa abordagem foi possível identificar algumas mudanças na dinâmica das relações entre as microrregiões do estado após a implantação da regulação. As microrregiões que apresentam estabelecimentos de Alta Complexidade em Oncologia exibiram um incremento no número de internações no segundo ano estudado. Observa-se ainda que a microrregião Rio de Janeiro mantém centralidade de grau nos dois momentos. A utilização da ARS para a avaliação de políticas públicas pode trazer uma importante contribuição para planejamento e gestão em saúde.

Rede Social; Neoplasias; Regulação e Fiscalização em Saúde

\author{
Correspondência \\ C. B. Carroll \\ Rua Uruguai 135, bloco 1, apto. 503, Rio de Janeiro, RJ \\ 20541-145, Brasil. \\ cibele.carroll@gmail.com \\ 1 Instituto de Estudos em Saúde Coletiva, Universidade Federal \\ do Rio de Janeiro, Rio de Janeiro, Brasil. \\ 2 Instituto Nacional de Câncer José Alencar Gomes da Silva, Rio \\ de Janeiro, Brasil.
}




\section{Introdução}

De acordo com a Portaria no 2.439, de dezembro de 2005 1, documento pelo qual foi instituída a Política Nacional de Atenção Oncológica (PNAO), a alta complexidade é apontada como componente fundamental desta última. Apresenta como atribuições: garantir acesso de pacientes com diagnóstico clínico ou histopatológico de câncer a esse nível de atenção, realizar estadiamento, prover tratamento e cuidado, e assegurar qualidade conforme rotinas estabelecidas. Tais funções seriam desempenhadas pelas Unidades de Assistência de Alta Complexidade em Oncologia (UNACON) e pelos Centros de Assistência de Alta Complexidade em Oncologia (CACON) 1,2.

A UNACON é o estabelecimento que fornece tratamento de oncologia clínica, cirurgia oncológica e paliação para os cânceres mais prevalentes no Brasil. Caso não tenha serviço de radioterapia, deve haver referência formal de tal modalidade terapêutica para seus usuários. Já o CACON é aquele que atende todos os tipos de câncer, oferecendo também radioterapia, mas não tem, obrigatoriamente, tratamento para crianças e adolescentes 1,2 .

Embora a Portaria no 2.439 tenha sido revogada em favor da Portaria no 874, de maio de 2013 , que implementou a Política Nacional para a Prevenção e Controle do Câncer na Rede de Atenção à Saúde das Pessoas com Doenças Crônicas no âmbito do Sistema Único de Saúde (SUS), as obrigações dos estabelecimentos de alta complexidade permanecem semelhantes, sendo apresentadas de forma mais detalhada neste último documento 3 .

A partir de 2013, foram incluídos nesse nível de atenção os Hospitais Gerais com Cirurgia Oncológica (HGCO). Esses realizam o tratamento cirúrgico do câncer para posterior encaminhamento do paciente, caso seja necessária terapia complementar ao procedimento cirúrgico, com quimioterapia, radioterapia ou tratamento de medicina nuclear. Esse encaminhamento deve ser feito por intermédio de regulação, para local que forneça terapia especializada 3 .

Desse modo, muitos hospitais públicos e alguns hospitais privados, em parceria com o SUS, estão capacitados para o tratamento de câncer de forma integral. Corroborando tal informação, a Portaria no 458, de fevereiro de 2017 4, apresenta os estabelecimentos habilitados em Alta Complexidade em Oncologia no Brasil.

O conceito de atendimento oncológico baseado na atuação de centrais de regulação foi inicialmente apresentado pela Portaria no 1.478, de dezembro de 1999 5, que determinava que as secretarias de saúde, municipais e estaduais, criassem tal órgão regulador em suas respectivas áreas de atuação. Na política vigente desde 2013, o "componente regulação" é abordado como parte da estrutura organizacional da rede de atenção à saúde, e desempenha a função de gerenciar a articulação entre os componentes da rede assistencial. Dessa forma, é responsável por definir os fluxos de atendimento, visando à garantia de equidade e à otimização de recursos 3.

$\mathrm{O}$ atendimento pelo sistema público destinado aos tumores sólidos, no Estado do Rio de Janeiro, passa obrigatoriamente pela Central de Regulação. O caminho a ser percorrido tem início na atenção básica ou em locais de atendimento de média complexidade para que, em seguida, após acionado, o órgão regulador encaminhe o indivíduo enfermo ao cuidado específico.

Com essa estrutura pretende-se organizar o atendimento oncológico, orientando o tratamento de acordo com a disponibilidade de vaga, local de residência do paciente e complexidade do caso, melhorando o aproveitamento da capacidade da rede de assistência. A conformação também objetiva diminuir o tempo de espera para o tratamento e evitar grandes deslocamentos para acesso ao cuidado 6 .

No Rio de Janeiro, a Central de Regulação iniciou suas atividades em junho de 2015, sob gestão estadual, organizando procedimentos ambulatoriais de alta complexidade. A unidade gerencia processos e especialidades com base em acordos com as secretarias municipais e estadual 7 .

Entendendo a atuação da regulação como ferramenta importante para a promoção de equidade e descentralização do cuidado, o estudo do deslocamento de pacientes para tratamento de câncer, antes e após este marco, pode fornecer subsídios para a melhoraria da gestão da assistência oncológica no Rio de Janeiro.

O mapeamento de fluxo origem/destino de pessoas, considerando informações sobre localização geográfica, já vem sendo usado em pesquisas em saúde com o objetivo de identificar possíveis lacunas assistenciais ou, ainda, conquistas quanto à descentralização e regionalização do cuidado. Esses estu- 
dos utilizam conceitos de organização e agrupamento hierárquico de localidades, conforme o volume e a direção de fluxos de bens, indivíduos ou comunicação 8,9 .

O presente trabalho avalia o fluxo de pacientes com câncer do aparelho digestivo, entre residência e local de internação, utilizando a teoria da Análise de Redes Sociais (ARS). Na ARS, o foco está no estudo das relações entre diferentes atores, identificando a dinâmica das interações entre os componentes de uma determinada rede de relações. Com essa abordagem, pretende-se trazer à luz quem se comunica com quem, centralidades, atores isolados, dentre outras medidas próprias a esse tipo de análise.

Pesquisas recentes têm utilizado ARS em saúde, investigando a existência de ligações preconizadas, ou não, entre os componentes da rede de atenção, para a avaliação de programas e estratégias de atenção à saúde, com resultados promissores 10,11. Esse tipo de análise amplia a compreensão acerca do cenário real onde são implementados e desenvolvidos os programas e as ações de saúde 12 .

Assim, esta pesquisa tem como objetivo analisar o deslocamento para tratamento de pessoas com tumores digestivos no Estado do Rio de Janeiro, antes e após a atuação da regulação estadual, sob a perspectiva da análise de redes sociais.

\section{Material e métodos}

\section{Desenho e população do estudo}

Foi desenvolvido um estudo ecológico analítico longitudinal, comparando os períodos anterior e posterior à atuação da regulação no Estado do Rio de Janeiro. Foram utilizados dados secundários, provenientes do Departamento de Informática do SUS (DATASUS), disponíveis na página de Internet http://www2.datasus.gov.br/DATASUS/index.php?area=0203\&id=6926 (acessada em 29/Set/2017), referentes ao Subsistema de Informações Hospitalares (SIH/SUS). Nessa página, constam os dados dos atendimentos hospitalares, segundo o principal diagnóstico da internação, assim como o local de residência (origem) e de internação (destino).

As microrregiões do Instituto Brasileiro de Geografia e Estatística (IBGE) foram usadas como unidade geográfica de análise visando a caracterizar os movimentos dentro das, bem como entre as diferentes Regiões de Saúde (RS) do estado; sendo estas últimas as unidades utilizadas pelos programas estaduais para o planejamento das ações em saúde.

Desse modo, as internações por microrregiões (IBGE) foram usadas como indicador do local de onde o indivíduo faz o tratamento oncológico e relacionadas às microrregiões de residência referentes aos casos levantados. A tabulação dos dados foi feita utilizando-se o programa TabWin 4.15 (http://www2.datasus.gov.br/DATASUS/index.php?area=060805), para os anos de 2013 e 2016, antes e após a atuação da central de regulação, respectivamente.

Foram incluídas as admissões realizadas nos hospitais conveniados com o SUS do Estado do Rio de Janeiro de indivíduos residentes no mesmo estado, vinculadas aos cânceres de esôfago, estômago, intestino delgado, cólon e reto, canal anal, fígado, vesícula e vias biliares, pâncreas e neoplasias malignas de localizações mal definidas no aparelho digestivo (Classificação Internacional de Doenças, 10a revisão - CID-10: C15, C16, C17, C18, C19, C20, C21, C22, C23, C24, C25, C26).

A quantificação dos estabelecimentos de alta complexidade por microrregião do estado foi feita com o auxílio da Portaria no 458/2017 4, na qual estão listados todos os estabelecimentos habilitados em oncologia no país que oferecem tratamento pelo SUS. Todas as unidades no Instituto Nacional de Câncer José Alencar Gomes da Silva (INCA) foram contadas como 1 CACON.

As internações para pacientes com idade inferior a 20 anos foram excluídas da análise, uma vez que a regulação de tratamento oncológico pediátrico apresenta particularidades. Do mesmo modo, não foram contabilizados os hospitais de alta complexidade especializados no tratamento de câncer infantil. Por se tratar de estudo sobre neoplasia maligna do trato digestivo, as unidades especializadas em doenças exclusivamente hematológicas também não foram incluídas.

Assim, as 18 microrregiões do estado foram listadas e categorizadas em contentoras de hospital geral, UNACON e CACON, na dependência do tipo de atendimento mais complexo disponível para o tratamento oncológico (Quadro 1). 


\section{Quadro 1}

Microrregiões organizadas como nós, categorizadas pela complexidade do atendimento disponível.

\begin{tabular}{|c|c|c|c|}
\hline ID & MICRORREGIÃO & TIPO * & QUANTIDADE DE ESTABELECIMENTOS DE ALTA COMPLEXIDADE EM ONCOLOGIA ** \\
\hline $\mathrm{n} 1$ & Itaperuna & UNACON & 1 UNACON \\
\hline $\mathrm{n} 2$ & Santo Antônio de Pádua & Hospital Geral & 0 \\
\hline n3 & Campos dos Goytacazes & UNACON & 3 UNACONS \\
\hline $\mathrm{n} 4$ & Macaé & Hospital Geral & 0 \\
\hline n5 & Três Rios & Hospital Geral & 0 \\
\hline n6 & Cantagalo/Cordeiro & Hospital Geral & 0 \\
\hline $\mathrm{n} 7$ & Nova Friburgo & Hospital Geral & 0 \\
\hline n8 & Santa Maria Madalena & Hospital Geral & 0 \\
\hline n9 & Bacia de São João & Hospital Geral & 0 \\
\hline $\mathrm{n} 10$ & Lagos & UNACON & 1 UNACON \\
\hline $\mathrm{n} 11$ & Vale do Paraíba & UNACON & 2 UNACONS \\
\hline $\mathrm{n} 12$ & Barra do Piraí & Hospital Geral & 0 \\
\hline $\mathrm{n} 13$ & Baía da Ilha Grande & Hospital Geral & 0 \\
\hline $\mathrm{n} 14$ & Vassouras & UNACON & 1 UNACON \\
\hline $\mathrm{n} 15$ & Serrana & UNACON & 2 UNACONS \\
\hline $\mathrm{n} 16$ & Macacu/Caceribu & UNACON & 1 UNACON \\
\hline $\mathrm{n} 17$ & Itaguaí & Hospital Geral & 0 \\
\hline $\mathrm{n} 18$ & Rio de Janeiro & CACON & 2 CACONs, 9 UNACONs, 2 HGCOs \\
\hline
\end{tabular}

CACON: Centros de Assistência de Alta Complexidade em Oncologia ; HGCO: Hospitais Gerais com Cirurgia Oncológica; ID: identificação dos nós; UNACON: Unidades de Assistência de Alta Complexidade em Oncologia.

Fonte: elaboração dos autores.

* Atendimento mais complexo disponível em oncologia;

** Estabelecimentos de alta complexidade disponíveis para internação de pacientes com tumores do aparelho digestivo.

\section{Organização de dados para análise de redes sociais}

A organização dos dados para análise de redes foi feita por meio da construção de uma matriz de adjacências relacionando a microrregião de residência (linha) com a de internação (coluna). A matriz de adjacências consiste em uma matriz $n x n$, na qual o número 1 representa a ligação entre duas microrregiões, e 0 significa a ausência de relação entre elas 13. Com a intenção de atribuir intensidade às relações, foi criada uma matriz de pesos. Nesse caso, quanto maior o valor no interior das células da matriz, mais forte é a relação estabelecida. Neste estudo, os valores na matriz de pesos indicam o número de internações de pacientes residentes na microrregião $n \mathrm{x}$ (linha) que foram internados em hospitais da ny (coluna). Para a análise dos períodos pré e pós-atuação da regulação, foram criadas duas matrizes: uma com dados de 2013 e outra de 2016.

Partindo da matriz, é possível a formação dos grafos, que consistem em conjuntos de vértices, conectados por arestas (ligações sem direcionalidade) ou arcos (caso haja direção nas relações estabelecidas). Em ARS, os vértices correspondem a indivíduos, instituições, organizações, dentre outros, sendo chamados de Nós ou Atores, e o grafo passa a ser chamado de Sociograma 14.

As arestas e arcos evidenciam a troca de recursos entre os atores. Por se tratar de uma rede social, os recursos intercambiados são chamados de capital social, podendo ser dos mais variados tipos: financeiros, informações, cuidados 14 . No estudo em questão, trata-se da quantidade de admissões hospitalares, relacionando local de residência e destino para internação. Essa representação gráfica permite a visualização da estrutura das relações de um determinado grupo. Para a confecção dos 
sociogramas foi utilizado o programa Gephi versão 0.9.1 (https://gephi.org/users/download/), de livre acesso 15 .

Foram, assim, criados dois sociogramas das relações existentes entre as 18 microrregiões do Estado do Rio de Janeiro. Na apresentação gráfica, cada nó é representado por um círculo e identificado como a letra $\mathrm{n}(\mathrm{n} 1, \mathrm{n} 2, \mathrm{n} . .$.$) , conforme codificado no Quadro 1. Nesta pesquisa, a área do círculo é$ proporcional ao grau de entrada, representando o total de internações ocorridas nas microrregiões.

Os arcos apresentam diferentes espessuras, demonstrando o peso ou intensidade do intercâmbio de internações. Nos gráficos gerados pelo programa Gephi, a curvatura do arco aponta para a esquerda de onde o fluxo parte, ou seja, para esquerda da origem 15 . Por esse motivo, e visando a facilitar a compreensão do gráfico, as pontas de seta foram suprimidas sem prejuízo da interpretação.

No caso de a internação ocorrer dentro da mesma microrregião de residência, observa-se uma "self loop", ou autoligação. Nesta pesquisa, "self loops" estão expressas como figura ("etiqueta") acoplada aos nós, cujos tamanhos correspondem proporcionalmente ao volume de admissões hospitalares de residentes na própria microrregião.

Nos sociogramas deste estudo, as microrregiões que apresentam hospitais gerais são representadas por círculos em azul, UNACON em laranja e CACON em verde. Os arcos apresentam a mesma cor da microrregião de origem.

\section{Métricas de rede utilizadas}

Todas as métricas de rede apresentadas na pesquisa foram calculadas pelo programa Gephi versão 0.9.1, e são descritas a seguir. A densidade da rede diz respeito à quantidade de ligações estabelecidas entre seus atores, em relação ao total de conexões possíveis. Expressa em proporção, apresenta valor máximo de um, quando todos os atores se conectam entre si, e mínimo de zero, caso nenhum nó se relacione com os demais. Já a centralidade de grau é uma das medidas de importância de um nó dentro da rede, sendo aferida como a quantidade de ligações diretas estabelecidas por um determinado ator. Caso todos os "nós" recebam o mesmo número de ligações, a centralidade de grau é 016.

$\mathrm{O}$ algoritmo de modularidade identifica os atores mais fortemente conectados entre si, formando pequenas comunidades ou clusters.

Por se tratar de um grafo dirigido, em que existe orientação na relação, pode-se observar o grau de entrada (in degree) e o grau de saída (out degree) dos diferentes atores da rede. O valor atribuído ao grau de entrada representa, aqui, o número de internações ocorridas na região; provenientes do próprio local ou de outros nós. Já o valor atribuído ao grau de saída indica o encaminhamento de pacientes para internação em local distinto da área de residência.

A métrica HITS (Hyperlink-Induced Topic Search) utiliza os graus de entrada e saída para classificar os atores com "Authority" e "Hub". Authority é aquele para o qual muitos arcos se dirigem, e Hub, por sua vez, é o nó que aponta para muitos outros, possuindo assim um alto grau de saída 15.

\section{Fluxo preconizado no Estado do Rio de Janeiro}

O Plano Diretor de Regionalização (PDR) representa um dos instrumentos de planejamento utilizado pelo Governo do Estado do Rio de Janeiro para a promoção da descentralização e regionalização de ações e serviços de saúde. Tem como objetivo garantir acesso dos cidadãos a todos os serviços necessários, para a solução dos problemas de saúde, em todos os níveis de atenção 17.

Considera as RS como os conjuntos de municípios que têm atenção primária, serviços de urgência e emergência, atenção psicossocial, atenção ambulatorial e hospitalar especializada, bem como vigilância em saúde. Além desses pré-requisitos estruturais, a RS é compreendida como o um "espaço geográfico contínuo constituído por agrupamentos de municípios limítrofes, delimitado a partir de identidades culturais, econômicas e sociais e de redes de comunicação e infraestrutura de transportes compartilhados..." 17. Assim, devido à proximidade e identidade comuns, o planejamento e as ações de saúde são voltados para essas unidades 17.

Com base nesse documento, compreende-se que as RS que apresentam Alta Complexidade em Oncologia são capazes de prover tratamento cirúrgico e paliação aos indivíduos portadores de tumores do trato digestivo residentes na respectiva localidade 17 . O deslocamento para tratamento fora de 
domicílio (TFD) é previsto pelo PDR, e está descrito especialmente para tratamento de quimioterapia, radioterapia e cirurgia de cabeça e pescoço.

O Estado do Rio de Janeiro apresenta nove RS que compreendem as 18 microrregiões do estudo: RS Noroeste contém as microrregiões Itaperuna (n1) e Santo Antônio de Pádua (n2); RS Norte comporta Campos dos Goytacazes (n3) e Macaé (n4); RS Centro-sul contém Três Rios (n5) e Vassouras (n14); RS Serrana compreende as microrregiões Cantagalo/Cordeiro (n6), Nova Friburgo (n7), Santa Maria Madalena (n8), Serrana (n15) e parte da microrregião Macacu/Caceribu (n16); RS Baixada Litorânea abrange parte de Bacia de São João (n9) e Lagos (n10); RS Médio Paraíba contém Vale do Paraíba (n11) e Barra do Piraí (n12); RS Baía da Ilha Grande compreende a microrregião homônima (n13); RS Metropolitana I e RS Metropolitana II juntas abrangem parte de Bacia de São João (n9), parte de Macacu/Caceribu (n16), Itaguaí (n17) e Rio de Janeiro (n18).

\section{Resultados}

O primeiro sociograma, apresentado na Figura 1, é formado com dados de 2013 e apresenta 18 nós com 68 arcos, densidade de 0,22. O segundo sociograma, Figura 2, traz informações de 2016, exibindo as mesmas 18 microrregiões conectadas por 71 arcos, mostrando um incremento discreto no número de conexões, densidade de 0,23 . Sete arcos saíram do sociograma e surgiram dez novos, ocasionando um aumento da quantidade total de ligações existentes na rede (apenas três).

Ainda com relação às métricas de rede, observa-se que a microrregião Rio de Janeiro apresenta centralidade de grau, tanto em 2013 quanto em 2016, uma vez que estabelece mais relações com os demais nós: no primeiro sociograma recebe pacientes de todas as microrregiões, já no segundo, deixa de receber pacientes de Itaperuna.

Por meio do algoritmo de modularidade, para a rede de 2013 o coeficiente encontrado foi de 0,51 e foram identificados 5 agrupamentos: (a) Itaperuna e Pádua; (b) Campos e Macaé; (c) Três Rios e Vassouras; (d) Vale do Paraíba, Barra do Piraí, Baía de Ilha Grande; (e) Cantagalo/Cordeiro, Nova Friburgo, Santa Maria Madalena, Bacia de São João, Lagos, Serrana, Macacu/Caceribu, Itaguaí, Rio de Janeiro. O coeficiente da rede de 2016 apresentou valor semelhante, 0,48, entretanto, foram identificadas 4 comunidades: (a) Itaperuna, Pádua, Campos, Macaé, Cantagalo/Cordeiro; (b) Três Rios e Vassouras; (c) Vale do Paraíba, Barra do Piraí; (d) Nova Friburgo, Santa Maria Madalena, Bacia de São João, Lagos, Baía de Ilha Grande, Serrana, Macacu/Caceribu, Itaguaí, Rio de Janeiro. As comunidades identificadas são apresentadas nas Figuras 3 e 4.

São descritos como Authority em 2013, em ordem decrescente: Rio de Janeiro, Itaperuna, Serrana, Campos, Lagos, Vassouras, Vale do Paraíba. Para o ano de 2016, a distribuição aconteceu de maneira um pouco diferente: Rio de Janeiro, Itaperuna, Serrana, Vassouras, Vale do Paraíba, Macaé, Campos.

Os três principais atores identificados como Hub em 2013 foram: Santa Maria Madalena, Macaé, Nova Friburgo. Em 2016, os três principais foram: Cantagalo/Cordeiro, Lagos e Nova Friburgo. Os resultados das métricas de rede usadas na pesquisa são apresentados na Tabela 1.

\section{Discussão}

Comparando os momentos anterior e posterior à atuação da central de regulação no Estado do Rio de Janeiro, podem ser percebidas algumas mudanças na dinâmica do fluxo entre residência e local de tratamento de pacientes com tumores do aparelho digestivo.

Pode-se observar um aumento do número de internações em microrregiões detentoras de Alta Complexidade em Oncologia, que, na análise de redes, foi evidenciado como um maior grau de entrada. Visualmente, o incremento das internações em microrregiões que têm UNACON é observado por meio do aumento do tamanho das etiquetas aderidas aos nós, representando o aumento das autoligações (internações de residentes locais), bem como pelo aumento das dimensões dos nós devido ao maior número de conexões a eles dirigidas (internações provenientes de outras microrregiões).

Particularmente as regiões Serrana e Vale do Paraíba, apresentaram um incremento no número de internações, oriundas de microrregiões vizinhas. A primeira passou a receber pacientes de Cantagalo 
Figura 1

Sociograma 2013.

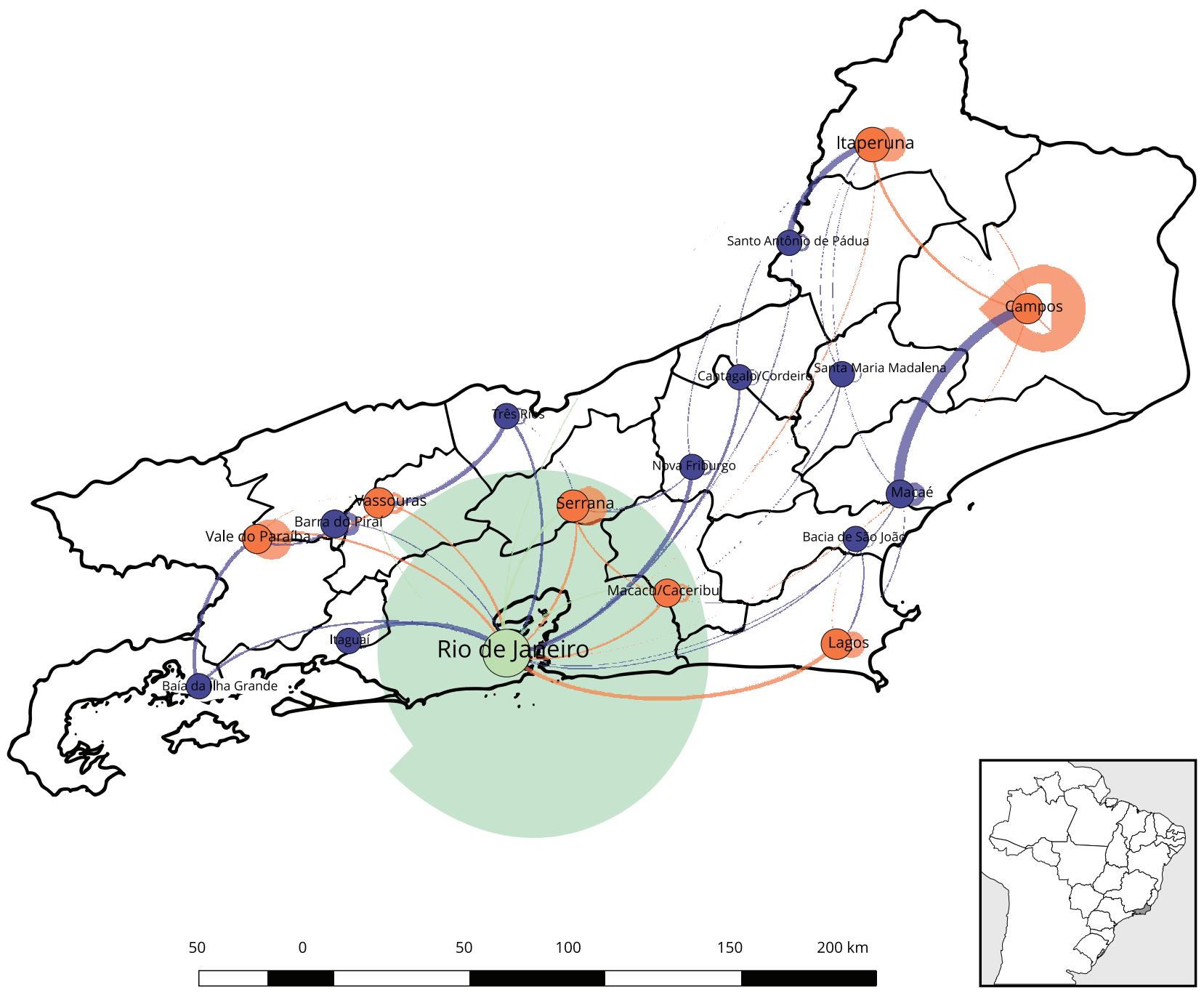

Fonte: elaborado pelos autores.

Nota: arcos com a cor do nó de origem, representando o fluxo de pacientes da área de residência para o local de internação. Etiqueta em torno do nó representando o quantitativo de internações locais de pacientes residentes na microrregião. Microrregião em azul: apresenta Hospital Geral; microrregião em laranja: apresenta Unidade de Assistência de Alta Complexidade em Oncologia (UNACON); microrregião em verde: apresenta Centro de Assistência de Alta Complexidade em Oncologia (CACON). Sociograma desenhado com o programa Gephi versão 0.9.1.

e a segunda intensificou a relação com Barra do Piraí. Essas relações são preconizadas pelo PDR de 2012/2013 17, uma vez que ocorrem dentro das respectivas RS. Os resultados da métrica Authority para o ano de 2016 também reforçam a aquisição de relevância do Vale do Paraíba, que passou a ser a quinta microrregião que mais recebeu internações no ano (houve aumento do grau de entrada). A habilitação de um novo UNACON em 2016, em Barra Mansa, pode ter contribuído para esse aumento das internações 18.

O melhor aproveitamento da capacidade da rede de assistência, por intermédio do aumento de internações em outras microrregiões que não a do Rio de Janeiro, parece ser um passo inicial no caminho da descentralização e da regionalização da assistência oncológica. 
Figura 2

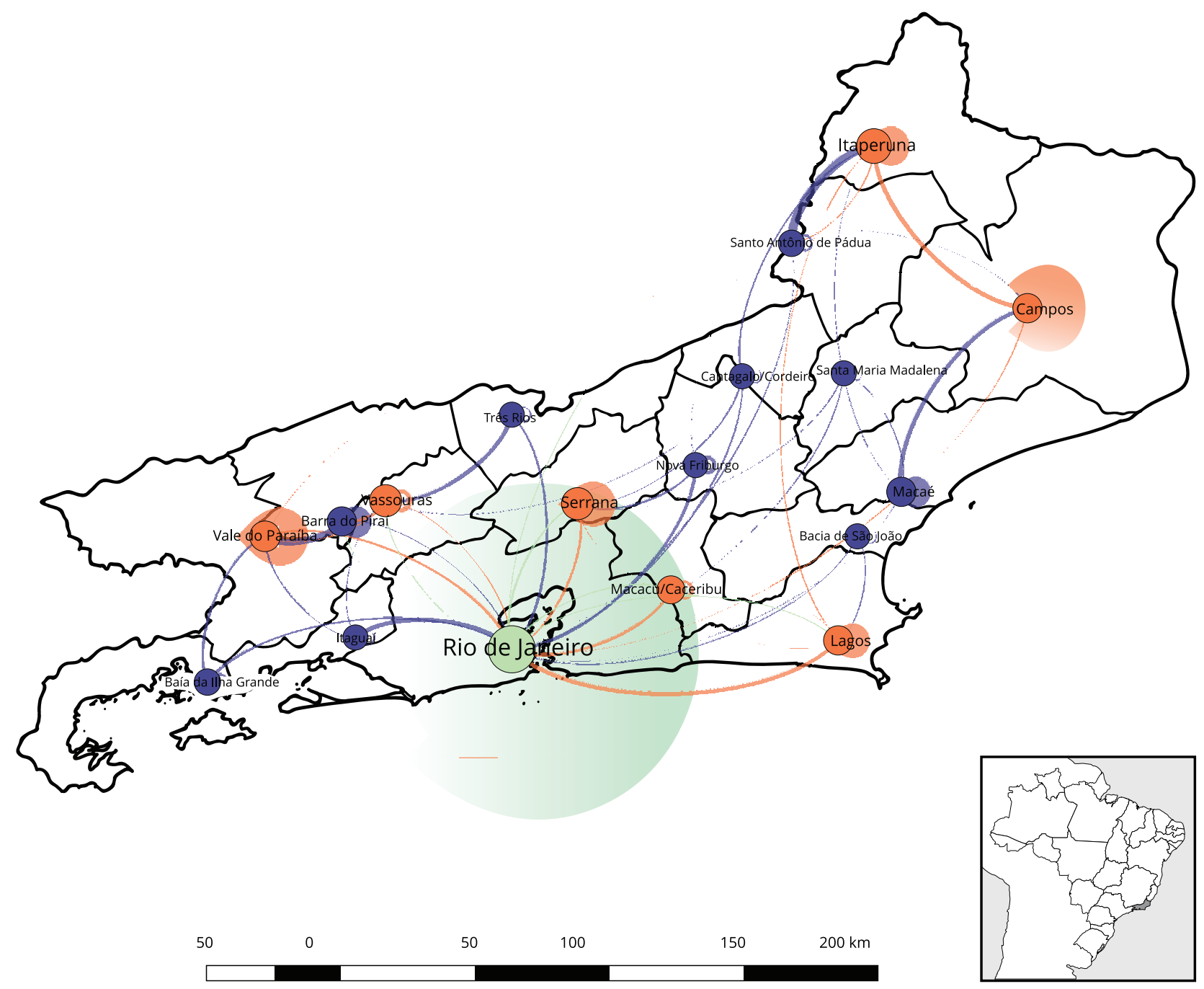

Fonte: elaborado pelos autores.

Nota: arcos com a cor do nó de origem, representando o fluxo de pacientes da área de residência para o local de internação. Etiqueta em torno do nó representando o quantitativo de internações locais de pacientes residentes na microrregião. Microrregião em azul: apresenta Hospital Geral; microrregião em laranja: apresenta Unidade de Assistência de Alta Complexidade em Oncologia (UNACON); microrregião em verde: apresenta Centro de Assistência de Alta Complexidade em Oncologia (CACON). Sociograma desenhado com o programa Gephi versão 0.9.1.

Considerando-se que a chegada aos serviços de saúde pode ser resultante tanto da postura do indivíduo frente à doença quanto das características da oferta de cuidado, a disponibilidade de tratamento próximo ao local de residência facilita o acesso dos pacientes. Num primeiro momento, favorece a entrada mais precoce do enfermo na assistência especializada, diminuindo o tempo de espera para o tratamento. Além disso, ao longo do acompanhamento terapêutico contribui para a adesão ao diminuir custos financeiros e psicossociais com deslocamento, alimentação, estadia, acompanhante e rede de suporte social; elementos que podem ser decisivos na continuidade do tratamento oncológico. Desse modo, o planejamento das políticas públicas deve ser capaz de identificar em quais locais a 


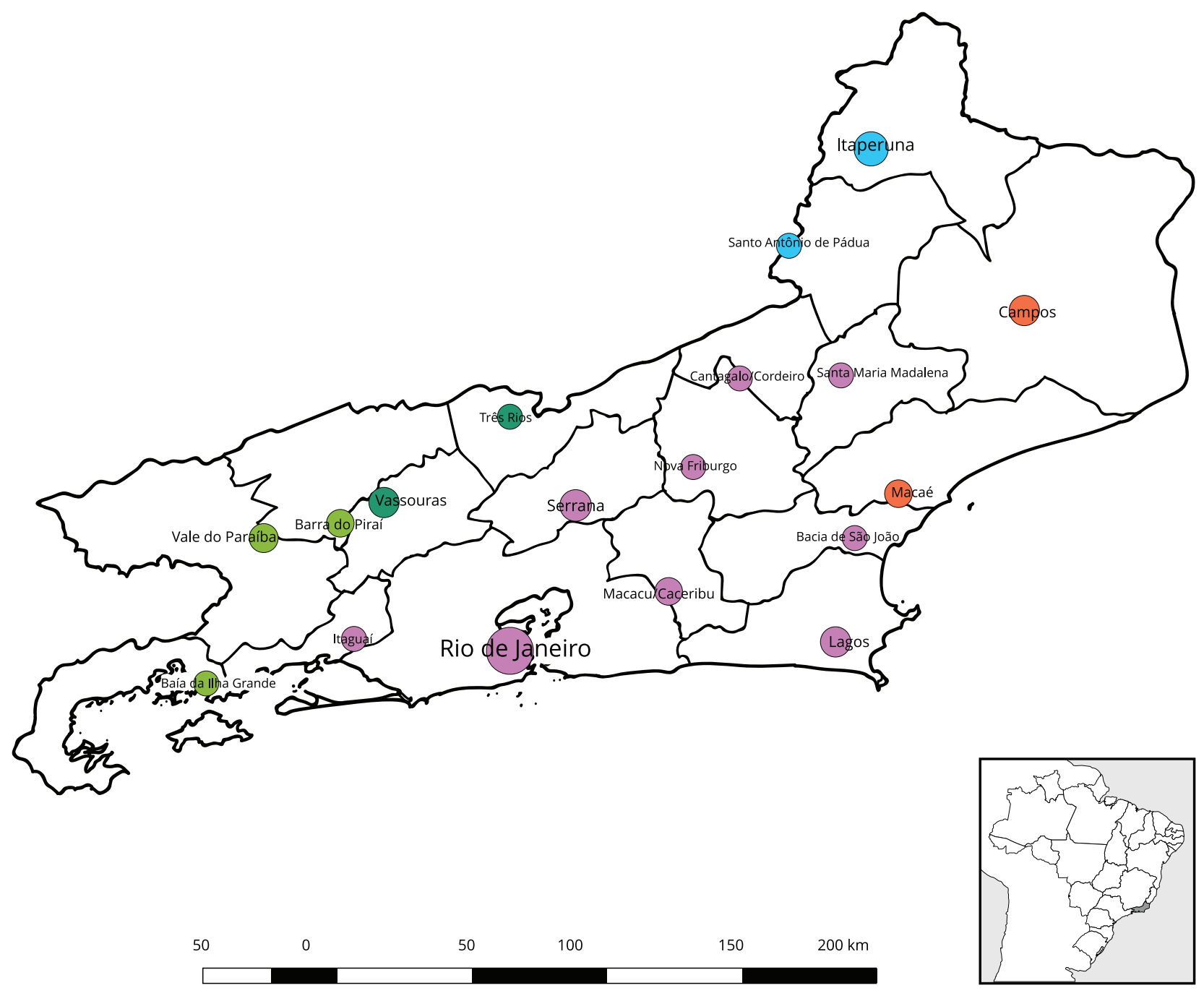

Fonte: elaborado pelos autores.

Nota: cinco comunidades identificadas no ano de 2013, diferenciadas pelas cores. Comunidades organizadas com o programa Gephi versão 0.9.1.

atenção parece mais ou menos favorável e interessante ao paciente, com o intuito de propor mudanças e adequações 8 .

Por outro lado, com exceção de Itaperuna e Campos que, pelos dados, parecem atuar de maneira praticamente autossuficiente quanto a seus pacientes oncológicos, ao apresentar baixo grau de saída, as outras microrregiões com Alta Complexidade em Oncologia ainda mantêm um percentual elevado de pacientes residentes que são internados fora do domicílio mesmo após a atuação da regulação.

Especialmente contraditória, a microrregião Lagos, que tem UNACON, aumentou o fluxo de pacientes para internação no Rio de Janeiro em paralelo a um considerável acréscimo de atendimento aos residentes locais. O Plano Estadual de Atenção Oncológica sugere que, com base na população residente na baixada litorânea, haveria necessidade de mais 1 UNACON/CACON nesta localidade, o que pode justificar o deslocamento de pacientes para tratamento fora do domicílio 18 . 
Figura 4

Comunidades identificadas em 2016.

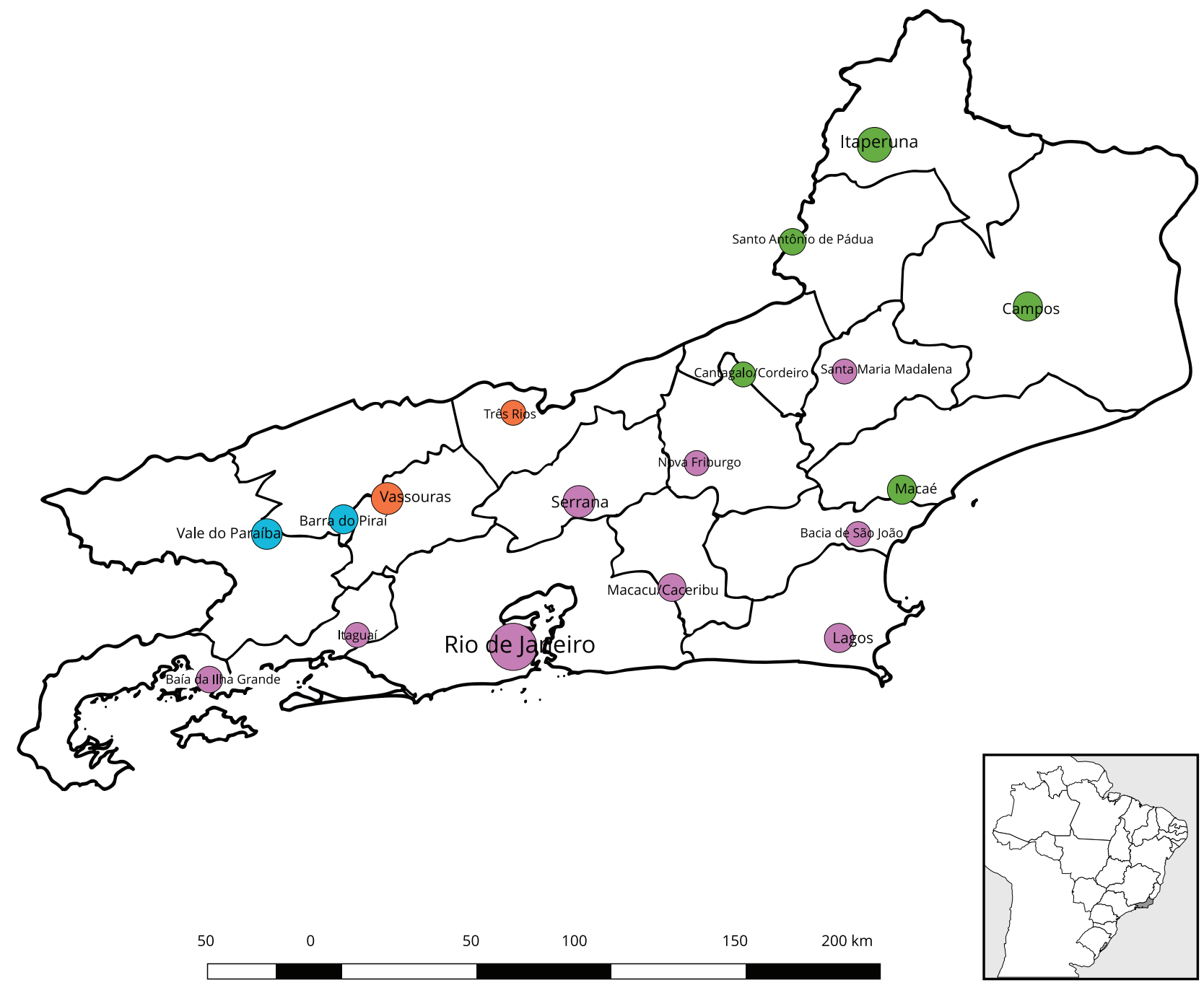

Fonte: elaborado pelos autores.

Nota: quatro comunidades identificadas no ano de 2016, diferenciadas pelas cores. Comunidades organizadas com o programa Gephi versão 0.9.1.

A posição de Hub, por apresentar um alto grau de saída, nesta pesquisa é mais comum para as regiões que têm apenas hospitais gerais, uma vez que devem obrigatoriamente encaminhar seus pacientes para tratamento onde o cuidado especializado se encontra disponível, conforme indicam as políticas norteadoras da atenção oncológica do estado 18.

Para os tumores do trato digestivo, quimioterapia e radioterapia são conduzidas em regime ambulatorial. Assim, pode-se inferir que a hospitalização por tais neoplasias acontece para cirurgia, manejo de paliação ou de efeitos colaterais de quimioterapia. Parece haver, aqui, um desafio a ser enfrentado no sentido que as regiões que contam com tratamento especializado absorvam ainda mais as internações da sua clientela residente para tratamento cirúrgico e paliativo.

Macaé, embora não conte com unidade especializada para tratamento de câncer listada como alta complexidade na Portaria $n^{\circ}$ 458/20174, exibe um acréscimo no número de internações para tratamen- 


\section{Tabela 1}

Resultados das métricas de rede utilizadas.

\begin{tabular}{|c|c|c|c|c|c|c|c|c|c|c|c|}
\hline \multirow[t]{2}{*}{ ID } & \multirow[t]{2}{*}{ Microrregião } & \multicolumn{2}{|c|}{$\begin{array}{c}\text { Centralidade de } \\
\text { grau * }\end{array}$} & \multicolumn{2}{|c|}{ Grau de entrada ** } & \multicolumn{2}{|c|}{ Grau de saída *** } & \multicolumn{2}{|c|}{ Authority \# } & \multicolumn{2}{|c|}{ Hub \#\# } \\
\hline & & 2013 & 2016 & 2013 & 2016 & 2013 & 2016 & 2013 & 2016 & 2013 & 2016 \\
\hline $\mathrm{n} 1$ & Itaperuna & 286 & 398 & 282 & 397 & 4 & 1 & 0,38 & 0,38 & 0,20 & 0,00 \\
\hline $\mathrm{n} 2$ & $\begin{array}{l}\text { Santo Antônio de } \\
\text { Pádua }\end{array}$ & 95 & 122 & 22 & 19 & 73 & 103 & 0,00 & 0,00 & 0,27 & 0,25 \\
\hline n3 & $\begin{array}{l}\text { Campos dos } \\
\text { Goytacazes }\end{array}$ & 1.016 & 916 & 994 & 873 & 22 & 43 & 0,22 & 0,14 & 0,23 & 0,23 \\
\hline n4 & Macaé & 176 & 227 & 83 & 179 & 93 & 48 & 0,08 & 0,14 & 0,32 & 0,25 \\
\hline n5 & Três Rios & 79 & 66 & 13 & 13 & 66 & 53 & 0,00 & 0,00 & 0,24 & 0,20 \\
\hline n6 & Cantagalo/Cordeiro & 23 & 31 & 7 & 1 & 16 & 30 & 0,00 & 0,00 & 0,23 & 0,33 \\
\hline n7 & Nova Friburgo & 66 & 92 & 17 & 41 & 49 & 51 & 0,00 & 0,00 & 0,28 & 0,28 \\
\hline n8 & $\begin{array}{l}\text { Santa Maria } \\
\text { Madalena }\end{array}$ & 8 & 7 & 2 & 1 & 6 & 6 & 0,00 & 0,00 & 0,33 & 0,23 \\
\hline n9 & Bacia de São João & 28 & 19 & 5 & 2 & 23 & 17 & 0,00 & 0,00 & 0,23 & 0,21 \\
\hline $\mathrm{n} 10$ & Lagos & 176 & 301 & 128 & 248 & 48 & 53 & 0,21 & 0,14 & 0,17 & 0,29 \\
\hline n11 & Vale do Paraíba & 392 & 655 & 358 & 620 & 34 & 35 & 0,11 & 0,17 & 0,19 & 0,20 \\
\hline $\mathrm{n} 12$ & Barra do Piraí & 135 & 309 & 99 & 219 & 36 & 90 & 0,08 & 0,13 & 0,21 & 0,23 \\
\hline $\mathrm{n} 13$ & Baía da Ilha Grande & 90 & 75 & 32 & 31 & 58 & 44 & 0,00 & 0,05 & 0,17 & 0,18 \\
\hline $\mathrm{n} 14$ & Vassouras & 130 & 132 & 95 & 92 & 35 & 40 & 0,18 & 0,25 & 0,19 & 0,21 \\
\hline $\mathrm{n} 15$ & Serrana & 301 & 402 & 283 & 387 & 18 & 15 & 0,27 & 0,27 & 0,15 & 0,15 \\
\hline $\mathrm{n} 16$ & Macacu/Caceribu & 88 & 83 & 59 & 54 & 29 & 29 & 0,09 & 0,11 & 0,25 & 0,23 \\
\hline $\mathrm{n} 17$ & Itaguaí & 55 & 73 & 1 & 7 & 54 & 66 & 0,00 & 0,00 & 0,15 & 0,23 \\
\hline $\mathrm{n} 18$ & Rio de Janeiro & 4.577 & 4.237 & 4.522 & 4.182 & 55 & 55 & 0,77 & 0,76 & 0,24 & 0,26 \\
\hline
\end{tabular}

ID: identificação dos nós.

Fonte: elaboração dos autores.

Nota: métricas de rede calculadas pelo programa Gephi versão 0.9.1.

* Total de arestas ligadas aos nós, valores ponderados pelos pesos; representam o total de internações vinculadas às microrregiões;

** Total de internações ocorridas nas microrregiões, incluindo residentes locais e provenientes das outras microrregiões;

valores ponderados pelos pesos;

*** Total de internações encaminhadas para outras microrregiões; valores ponderados pelos pesos;

\# Valores calculados com algoritmo HITS, classifica os nós de acordo com o grau de entrada;

\#\# Valores calculados com algoritmo HITS, classifica os nós de acordo com o grau de saída.

to de neoplasia do trato gastrointestinal após 2015. Em 2013, era o segundo ator com maior grau de saída. Já em 2016, apresentou coeficiente Authority semelhante ao de Campos dos Goytacazes que tem três UNACONs. Esse fato pode ter relação com uma iniciativa da prefeitura local, que instituiu o Plano de Atenção Oncológica Municipal, em 2013, por meio do estabelecimento de parcerias público-privadas, conforme informações no site da Prefeitura 19.

As internações de residentes locais na microrregião Rio de Janeiro foram superiores a $3.800 \mathrm{em}$ ambos os anos e, por isto, nos sociogramas, representados pela "etiqueta" acoplada ao nó, cobrem parte da representação gráfica. Durante os dois momentos estudados também foi o ator que mais recebeu arcos provenientes das demais microrregiões. Assim, manteve a centralidade de grau e apresentou coeficiente Authority mais elevado antes e após a regulação.

A manutenção da centralidade de grau, de maneira díspar, pode ser explicada pelo fato de conter mais da metade dos estabelecimentos de Alta Complexidade em Oncologia, associada à maior população do estado. Enquanto os demais 17 atores dividem 11 UNACONs, esse ator conta com 2 CACONs, 9 UNACONs e 2 HGCO, conforme consta na Portaria no 458/20174. 
Quanto à população alvo, o planejamento proposto pela Portaria no 140/2014 20 indica que, para o tratamento oncológico, o ideal preconizado é de um estabelecimento de alta complexidade para cada 500 mil habitantes. Nos casos das regiões Sul e Sudeste do Brasil, por apresentarem uma grande parcela da população com idade superior a 50 anos, admite-se a habilitação de centro de alta complexidade para local com número inferior de habitantes, desde que ocorram pelo menos 900 novos casos de câncer por ano (exceto câncer de pele não melanoma) 18. A estimativa de câncer do INCA para o biênio 2018/2019 indica que as regiões Sul e Sudeste do país concentram 70\% dos casos novos de câncer, e aponta para a expectativa de 42.620 casos novos/ano para o Estado do Rio de Janeiro 21.

Dessa forma, a microrregião do Rio de Janeiro, com população superior a 12 milhões habitantes, poderia comportar mais instituições desse tipo, conforme o Plano Estadual de Atenção Oncológica, de maio de 2017 18. Esse documento aponta para um déficit de 13 estabelecimentos de alta complexidade em oncologia nas regiões metropolitanas I e II 18. A existência de um déficit de estabelecimentos torna compreensível o fato de existir um êxodo de pacientes com neoplasia do trato digestivo para tratamento fora do respectivo local de residência. Esse fluxo para fora da microrregião que tem maior complexidade de cuidado e maior concentração de estabelecimentos, sugere que a microrregião atua acima de sua capacidade resolutiva.

No sociograma de 2013, foram identificadas cinco comunidades ou clusters, e no de 2016 foram encontradas quatro. A redução aconteceu por conta da fusão entre as díades Itaperuna/Santo Antônio de Pádua e Campos/Macaé. Assim, após a regulação, observa-se maior interação entre as RS Noroeste e Norte Fluminense que, conforme mencionado anteriormente, integradas, acabam por atuar próximo da autossuficiência, com poucas internações encaminhadas para o Rio de Janeiro. Uma associação que parece vantajosa quanto à regionalização.

A junção das RS Noroeste e Norte, formando uma comunidade na segunda avaliação de rede, é um bom exemplo de como as relações informais podem funcionar como uma estratégia de coesão social. Mesmo sem que haja o direcionamento formal do Plano Estadual de Atenção Oncológica, a norma social criada e gerida pelos indivíduos que compõem a rede estreitou o intercâmbio de pacientes de forma que se identificasse uma única comunidade onde inicialmente havia duas 22.

Já Nova Friburgo, Santa Maria Madalena, Serrana, Macacu/Caceribu, Bacia de São João, Lagos, Baía da Ilha Grande, Itaguaí e Rio de Janeiro estão fortemente conectados formando uma única comunidade em 2016. Entretanto, conforme o Plano Estadual de Atenção Oncológica, essas microrregiões fazem parte de cinco RS distintas e Baía da Ilha Grande poderia estar fortemente conectada ao Vale do Paraíba, local descrito como sua referência para cirurgia oncológica 17,18.

Desse modo, as relações dinâmicas que se estabelecem entre os diferentes atores da rede são capazes de indicar os desejos e interesses de determinado grupo; o que pode tanto ratificar as proposições políticas quanto apontar suas ineficiências e inadequações ao contexto social em que tal grupo está inserido 23,24.

Embora após a regulação tenha ocorrido intensificação dos laços entre alguns locais da mesma região de saúde, conforme preconizado pelo PDR e pela Política Estadual de Atenção Oncológica, ainda existem muitas relações entre as microrregiões que não obedecem ao planejado. No cenário real, muitos atores mantêm ligações fora da área determinada pelas políticas de saúde.

A rede desenhada após a implantação da regulação apresenta modificações discretas que favorecem a descentralização e regionalização do tratamento do câncer gastrointestinal, já que a maioria das microrregiões habilitadas para este tipo de cuidado obteve um incremento em sua atuação.

Diante dos dados, cabe o questionamento acerca das motivações para o fluxo diverso ao proposto pelas políticas de saúde. Além da insuficiência de estabelecimentos no Rio de Janeiro e na Baixada Litorânea, já descrita pelo Plano Estadual de Atenção Oncológica, é possível indagar se todas as microrregiões que têm alta complexidade estão realmente preparadas para o atendimento de sua população residente. Por outo lado, alguns dos locais de referência para o tratamento preconizado pelas políticas do estado podem não parecer atraentes para diferentes microrregiões pelos mais variados motivos como, por exemplo, distância geográfica e disponibilidade de transporte. Ao mesmo tempo, algumas relações não formais podem se mostrar vantajosas para a organização da rede.

A aplicação da ARS facilitou a compreensão acerca da dinâmica do deslocamento de pacientes com câncer do aparelho digestivo dentro do Estado do Rio de Janeiro. Mostrou também que, a despei- 
to das normas bem estabelecidas, os desdobramentos das políticas de saúde ocorrem em um contexto social sujeito às interferências dos diversos atores nele inseridos 25 .

O estudo apresenta limitações relacionadas ao fato de ter sido conduzido com dados secundários, podendo haver equívocos de digitação, subnotificação dos casos de câncer do aparelho digestivo, ou ainda, registros incorretos quanto ao local de residência. Contudo, por ter sido realizado com base de dados oficial, acredita-se que as informações reflitam o cenário real, sem comprometer o objetivo do trabalho.

Ainda sobre as limitações, como foram analisados apenas dois anos não é possível afirmar que as mudanças na rede formada pelas microrregiões do estado se devam exclusivamente à atuação da regulação. Além disso, o segundo ano estudado é o seguinte ao início das atividades da central estadual reguladora, o que pode prejudicar a avaliação dos efeitos da mesma. Desse modo, mais estudos acerca do tema são necessários.

\section{Conclusão}

O trabalho sugere que a participação da regulação para o atendimento às neoplasias malignas do trato gastrointestinal possa ter provocado mudanças na dinâmica das internações no Estado do Rio de Janeiro. À luz das redes desenhadas, observa-se um aumento do número de internações em microrregiões detentoras de estabelecimentos especializados para tal.

Mesmo com a regulação, ainda existe um grande quantitativo de pacientes que segue um fluxo diferente do preconizado pelos documentos norteadores da atenção ao câncer no estado, em especial para a microrregião Rio de Janeiro. O maior número de instituições especializadas nessa microrregião, bem como a presença da maior população no mesmo local, parecem ser alguns dos fatores responsáveis pela manutenção da centralidade dessa localidade.

A abordagem do tema sob a perspectiva da ARS parece ser uma estratégia metodológica eficaz, ao permitir a visualização dos atores e suas relações, o que a torna uma ferramenta prática para planejamento e gestão.

\section{Colaboradores}

Ambas as autoras participaram igualmente da concepção do projeto, análise e interpretação de dados. Ambas as autoras contribuíram para a redação do artigo, revisão do conteúdo intelectual e aprovação da versão final.

\section{Informações adicionais}

ORCID: Cibele Barbosa Carroll (0000-0003-26810928); Marcia Gomide (0000-0001-8364-4482).

\section{Agradecimentos}

Agradecemos ao colega Edmar Lopes pelas valiosas conversas sobre a rede de atenção oncológica. Agradecemos à Beatriz Cordeiro Jardim pelo apoio nas pesquisas com DATASUS. Agradecemos também à Kimberly Brown pelo design gráfico.

\section{Referências}

1. Ministério da Saúde. Portaria no 2439, de 8 de dezembro de 2005. Institui a Política Nacional de Atenção Oncológica: Promoção, Prevenção, Diagnóstico, Tratamento, Reabilitação e Cuidados Paliativos, a ser implantada em todas as unidades federadas, respeitadas as competências das três esferas de gestão. Diário Oficial da União 2005; 9 dez.

2. Ministério da Saúde. Portaria no 741, de 19 de dezembro de 2005. Define as unidades de assistência de alta complexidade em oncologia, os centros de assistência de alta complexidade em oncologia (CACON) e os centros de referência de alta complexidade em oncologia e suas aptidões e qualidades. Diário Oficial da União 2005; 23 dez.

3. Ministério da Saúde. Portaria no 874, de 16 de maio de 2013. Institui a Política Nacional para a Prevenção e Controle do Câncer na Rede de Atenção à Saúde das Pessoas com Doenças Crônicas no âmbito do Sistema Único de Saúde (SUS). Diário Oficial da União 2013; 17 mai. 
4. Ministério da Saúde. Portaria no 458, de 24 de fevereiro de 2017. Mantém as habilitações de estabelecimentos de saúde na Alta Complexidade e exclui prazo estabelecido na Portaria no 140/SAS/MS, de 27 de fevereiro de 2014. Diário Oficial da União 2017; 7 mar.

5. Ministério da Saúde. Portaria no 1.478 , de 28 de dezembro de 1999. Implanta as centrais de Programação e Regulação de Assistência Oncológica. Diário Oficial da União 1999; 29 dez.

6. Regular para garantir acesso. Rede Câncer 2010; (10):36-8.

7. Central Unificada de Regulação é inaugurada no Rio de Janeiro. http://www.brasil.gov.br/ editoria/saude/2015/06/central-unificadade-regulacao-e-inaugurada-no-rio-de-janeiro (acessado em 21/Set/2017).

8. Oliveira EXG, Melo ECP, Pinheiro RS, Noronha $\mathrm{CP}$, Carvalho MS. Acesso à assistência oncológica: mapeamento dos fluxos origemdestino das internações e dos atendimentos ambulatoriais. O caso do câncer de mama. Cad Saúde Pública 2011; 27:317-26.

9. Nystuen JD, Dacey MF. A graph theory interpretation of nodal regions. Pap Reg Sci 1961; 7:29-42.

10. Costa PH, Ronzani TM, Colugnati FA. Análise de redes sociais: uma estratégia avaliativa para a rede de atenção aos usuários de álcool e outras drogas. In: Ronzani TM, Sartes LM, Perucchi J, organizadores. Psicologia e saúde pública: contribuições e desafios. Juiz de Fora: Editora UFJF; 2015. p. 227-46.

11. Bean DM, Stringer C, Beeknoo N, Teo J, Dobson RJB. Network analysis of patient flow in two UK acute care hospitals identifies key subnetworks for A\&E performance. PLoS One 2017; 12:e0185912.

12. Lino C, Gomide M. Análise de redes sociais na avaliação do programa de controle de hanseníase em um município do interior do Brasil. Cad Saúde Colet (Rio J.) 2012; 20:32-40.

13. Zweig KA. Network analysis literacy: a practical approach to the analysis of networks. Vienna: Springer-Verlag; 2016.

14. Portugal S. Contributos para uma discussão do conceito de rede na teoria sociológica. Coimbra: Universidade de Coimbra; 2007. (Oficina do CES, 271).

15. Bastian M, Heymann S, Jacomy M. Gephi: an open source software for exploring and manipulating networks. In: Proceedings of the Third International ICWSM Conference. https:// www.aaai.org/ocs/index.php/ICWSM/09/pa per/view/154 (acessado em 30/Set/2017).
16. McCarty C. Structure in personal networks. J Soc Struct 2002; 3(1). https://www.cmu.edu/ joss/content/articles/volume3/McCarty.html.

17. Secretaria de Saúde. Atualização do Plano Diretor de Regionalização 2012/2013. https:// www.saude.rj.gov.br/comum/code/Mostra rArquivo.php?C=MzA0OQ\%2C\%2C (acessado em 01/Out/2017).

18. Secretaria de Saúde. Plano Estadual de Atenção Oncológica. Rio de Janeiro: Secretaria de Saúde; 2017.

19. Secretaria de Saúde. Tratamento humanizado aos pacientes com câncer completa dois anos. http://www.macae.rj.gov.br/saude/leitura/no ticia/tratamento-humanizado-aos-pacientescom-cancer-completa-dois-anos (acessado em 30/Set/2017).

20. Ministério da Saúde. Portaria no 140, de 27 de fevereiro de 2014. Redefine os critérios e parâmetros para organização, planejamento, monitoramento, controle e avaliação dos estabelecimentos de saúde habilitados na atenção especializada em oncologia e define as condições estruturais, de funcionamento e de recursos humanos para a habilitação destes estabelecimentos no âmbito do Sistema Único de Saúde (SUS). Diário Oficial da União 2014; 28 fev.

21. Instituto Nacional de Câncer José Alencar Gomes da Silva. Estimativa 2018: incidência de câncer no Brasil. Rio de Janeiro: Instituto Nacional de Câncer José Alencar Gomes da Silva; 2017.

22. Granovetter MS. The strength of weak ties. Am J Sociol 1973; 78:1360-80.

23. Lazega E. Redes e regulação: por um institucionalismo neo estrutural. Política \& Trabalho 2018; (48):217-25

24. Gomide M, Schütz GE. Análise de redes sociais e práticas avaliativas: desafios à vista. Physis (Rio J.) 2015; 25:819-42.

25. Blanchet K, James P. How to do (or not to do) a social network analysis in health systems research. Health Policy Plan 2012; 27:438-46. 


\section{Abstract}

High complexity is a fundamental component of Brazil's National Policy for Cancer Prevention and Control under the Unified National Health System (SUS). The policy mandates guaranteeing comprehensive patient care. Regulation is part of the organizational structure and is responsible for defining treatment flows. In Rio de Janeiro, the Central Regulating Office launched its activities in June 2015, organizing high-complexity outpatient procedures. The current study aims to analyze commuting for treatment by individuals with gastrointestinal tumors in the state of Rio de Janeiro, before and after the implementation of regulation, from the perspective of Social Network Analysis. This ecological study compared the periods before (2013) and after (2016) implementation of the Central Regulating Office. The study drew on secondary data from the Brazilian Health Informatics Department. Two sociograms were designed for the years 2013 and 2016, correlating place of residence with place of hospitalization. This approach allowed identifying some changes in the dynamics of relations between the state's microregions after implementation of the regulation. The microregions with high-complexity oncology establishments displayed an increase in the number of hospitalizations in 2016. The microregion of Rio de Janeiro also maintained degree centrality in the two moments. The use of Social Network Analysis to assess public policies can contribute to health planning and management.

Social Networking; Neoplasms; Health Care Coordination and Monitoring

\section{Resumen}

La alta complejidad es un componente fundamental de la Politica Nacional para la Prevención y Control del Cáncer en el ámbito del Sistema Único de Salud (SUS) brasileño. Tiene como obligación garantizar el cuidado integral a los pacientes. La regulación es parte de la estructura organizacional, siendo responsable de definir los flujos de atención. En Río de Janeiro, la Central de Regulación inició sus actividades en junio de 2015, organizando procedimientos ambulatorios de alta complejidad. El presente trabajo tiene como objetivo analizar el desplazamiento para el tratamiento de personas con tumores digestivos en el Estado de Río de Janeiro, antes y después de la actuación de la regulación, desde la perspectiva del Análisis de Redes Sociales (ARS). Se desarrolló un estudio ecológico, comparando los períodos anterior (2013) y posterior (2016) a la implementación de la central reguladora. La investigación fue desarrollada con la utilización de datos secundarios provenientes del Departamento de Informática del SUS. De este modo, se diseñaron dos sociogramas referentes a los años 2013 y 2016, relacionando lugar de residencia con local de internamiento. Con este abordaje fue posible identificar algunos cambios en la dinámica de las relaciones entre las microrregiones del Estado tras la implementación de la regulación. Las microrregiones que presentan establecimientos de Alta Complejidad en Oncología mostraron un incremento en el número de internamientos en el segundo año estudiado. Se observa incluso que la microrregión Río de Janeiro mantiene centralidad de nivel/grado en los dos momentos. La utilización de la ARS para la evaluación de políticas públicas puede conllevar una importante contribución para la planificación y gestión en salud.

Red Social; Neoplasias; Regulación y

Fiscalización en Salud
Recebido em 03/Mar/2018

Versão final reapresentada em 15/Mai/2019

Aprovado em 26/Jun/2019 